\title{
Pulmonary ultrasound findings in a bottlenose dolphin Tursiops truncatus population
}

\author{
Cynthia R. Smith ${ }^{1, *}$, Mauricio Solano ${ }^{2}$, Betsy A. Lutmerding ${ }^{1}$, Shawn P. Johnson ${ }^{1}$, \\ Jennifer M. Meegan ${ }^{1}$, Carolina R. Le-Bert ${ }^{1}$, Forrest Emory-Gomez ${ }^{1}$, \\ Stephen Cassle ${ }^{3,4}$, Kevin Carlin ${ }^{1}$, Eric D. Jensen ${ }^{3}$ \\ ${ }^{1}$ National Marine Mammal Foundation, San Diego, California 92106, USA \\ ${ }^{2}$ Cummings School of Veterinary Medicine, Tufts University, North Grafton, Massachusetts 01536, USA \\ ${ }^{3}$ US Navy Marine Mammal Program, SSC PACIFIC Code 71510, San Diego, California 92152, USA \\ ${ }^{4}$ Present address: College of Veterinary Medicine, University of Florida, Gainesville, Florida 32603, USA
}

\begin{abstract}
Lung disease is common among wild and managed populations of bottlenose dolphins Tursiops truncatus. The purpose of the study was to apply standardized techniques to the ultrasound evaluation of dolphin lungs, and to identify normal and abnormal sonographic findings associated with pleuropulmonary diseases. During a 5 yr period (2005 to 2010), 498 non-cardiac thoracic ultrasound exams were performed on bottlenose dolphins at the Navy Marine Mammal Program in San Diego, California, USA. Exams were conducted as part of routine physical exams, diagnostic workups, and disease monitoring. In the majority of routine exams, no abnormal pleural or pulmonary findings were detected with ultrasound. Abnormal findings were typically detected during non-routine exams to identify and track disease progression or resolution; therefore, abnormal results are overrepresented in the study. In order of decreasing prevalence, abnormal sonographic findings included evidence of alveolar-interstitial syndrome, pleural effusion, pulmonary masses, and pulmonary consolidation. Of these findings, alveolar-interstitial syndrome was generally nonspecific as it represented several possible disease states. Pairing ultrasound findings with clinical signs was critical to determine relevance. Pleural effusion, pulmonary masses, and pulmonary consolidation were relatively straightforward to diagnose and interpret. Further diagnostics were performed to obtain definitive diagnoses when appropriate, specifically ultrasound-guided thoracocentesis, fine needle aspirates, and lung biopsies, as well as radiographs and computed tomography (CT) exams. Occasionally, post mortem gross necropsy and histopathology data were available to provide confirmation of diagnoses. Thoracic ultrasound was determined to be a valuable diagnostic tool for detecting pleural and pulmonary diseases in dolphins.
\end{abstract}

KEY WORDS: Cetacean $\cdot$ Diagnostic imaging $\cdot$ Lung disease $\cdot$ Pulmonary

\section{INTRODUCTION}

Thoracic ultrasound provides rapid diagnosis of pleural and pulmonary disease in veterinary hospitals and human emergency rooms. Specific techniques for diagnosing pleuritis, pleural effusion, pleural masses, pneumothorax, lung masses, and alveolar-interstitial diseases have been described for small animal (Stowater \& Lamb 1989, Tidwell 1998, Reichle \& Wisner 2000, Mattoon \& Nyland 2002, Saunders \& Keith 2004, Hecht 2008, Louvet \& Bourgeois 2008, Larson 2009), large animal (Rantanen 1981, 1986, Rantanen et al. 1981, Jung \& Bostedt 2004, Ramirez et al. 2004), and human patients (Lichtenstein 2007, 2009, Cardinale et al. 2009, 2010, Barillari \& Fioretti 2010, Sartori \& Tombesi 2010, Gar- 
gani 2011, Moore \& Copel 2011, Stefanidis et al. 2011). General descriptions of dolphin thoracic ultrasound exams have been published in marine mammal textbooks (Stone 1990, Brook et al. 2001). However, there are currently no peer-reviewed publications on thoracic ultrasound techniques or findings in dolphins.

A retrospective epidemiologic analysis of lung histopathology from the past $30 \mathrm{yr}$ (1980 to 2010) of US Navy Marine Mammal Program data showed that the most common pulmonary pathology was pneumonia (Venn-Watson et al. 2012), which is similar to findings of wild dolphin necropsies (Baker 1992, Di Guardo et al. 1995, Cornaglia et al. 2000, Bogomolni et al. 2010). In both wild and managed dolphins, bacterial pneumonias have been reported often (Cusick \& Bullock 1973, Sweeney \& Ridgway 1975, Diamond et al. 1979, Tangredi \& Medway 1980, Howard et al. 1983, Schulman et al. 1997, Miller et al. 1999, Dunn et al. 2001, Makara et al. 2007, St. Leger et al. 2009), as well as fungal (Carroll et al. 1968, Migaki et al. 1978, Gales et al. 1985, Joseph et al. 1986, Schulman et al. 1997, Jensen et al. 1998, Reidarson et al. 1998a,b, Miller et al. 2002, Elad et al. 2011), viral (Domingo et al. 1992, Lipscomb et al. 1994a,b, 1996, Moeller 2003, Di Guardo et al. 2005, Nollens et al. 2008, Venn-Watson et al. 2008), and parasitic etiologies of pneumonia (Woodard et al. 1969, Greenwood \& Taylor 1977, Inskeep et al. 1990, Dailey et al. 1991, Parsons et al. 2001, Bonar et al. 2007, Kuwamura et al. 2007, Fauquier et al. 2009). Reports of pneumonia often included findings of pleural effusion and pulmonary abscessation. The high prevalence of pneumonia in both wild and captive dolphins underscores the importance of standardized methods for the evaluation of pleural and pulmonary disease in these animals.

Bottlenose dolphin lung anatomy has been well described in the literature (Fiebiger 1916, Wislocki 1929, Green 1972, Simpson \& Gardner 1972, Piscitelli et al. 2010). Dolphin lungs extend cranially to about the first cervical vertebra and as far caudally as the second or third lumbar vertebrae. The lungs are divided into left and right lobes and are covered in smooth, opaque, elastic pleura measuring 1 to $2 \mathrm{~mm}$ in thickness (Fiebiger 1916, Wislocki 1929). The cranioventral and mid-ventral portions of each lung field are very thin where they overlay the heart. Lymphatic circulation from each lung lobe drains to a marginal lymph node, which is a solitary node located in the pleural space caudolateral to the heart against the diaphragm. Each node typically measures $5 \times 2 \times 2 \mathrm{~cm}$ in an adult dolphin (Cowan \& Smith 1999). Terminal airways are reinforced with carti- lage, and myoelastic sphincters are found surrounding terminal bronchioles and alveolar entrances (Simpson \& Gardner 1972). The alveoli can completely collapse at depth, forcing air into the reinforced air spaces, presumably for prevention of decompression sickness (Ridgway et al. 1969). Functionally, the lungs serve a dual purpose of respiration and buoyancy control; therefore, respiratory disease manifestations can include changes in buoyancy.

The purpose of this study was to apply standardized techniques to the ultrasound evaluation of dolphin lungs in a managed population of bottlenose dolphins, including ultrasound-guided techniques for sampling lung lesions. We also identified normal and abnormal sonographic findings associated with pleuropulmonary diseases in this species.

\section{MATERIALS AND METHODS}

\section{Medical records}

A prospective ultrasound study was performed over a 5 yr period (2005 to 2010). Electronic ultrasound records were created for 498 transthoracic ultrasound exams that were performed on 79 bottlenose dolphins cared for by the US Navy in San Diego, California, USA, ranging in age from $1 \mathrm{wk}$ to $51 \mathrm{yr}$. Exams were performed either as part of a full-body ultrasound exam or a targeted thoracic evaluation for one of the following reasons: (1) annual physical exam, (2) diagnostic workup, or (3) disease monitoring. Ultrasound findings were entered into an electronic medical database by the performing clinician.

\section{Ultrasound equipment}

Portable ultrasound units were utilized primarily to facilitate dolphin ultrasound exams in or adjacent to water, allowing the dolphin to remain in its natural environment. The portable units deployed in the field and used in the hospital included the Sonosite 180 Plus, Sonosite MicroMaxx, Sonosite M-Turbo, and GE Voluson i. For the Sonosite units, curvilinear transducers ranging from 2 to $5 \mathrm{MHz}$ were used. For the GE Voluson i, either a 2 to $5 \mathrm{MHz}$ 3D/4D transducer or a 2 to $5 \mathrm{MHz}$ curvilinear transducer was used. Field units were fitted with personal video heads-up displays (eMagin and Carl Zeiss). Transducers were routinely submerged in the water without probe covers and cleaned with ultrasound disinfectant-detergent solution. 


\section{Technique}

Ultrasound exams were performed as follows: (1) with the animal floating in an ocean enclosure and the sonographer, technician, and equipment on land
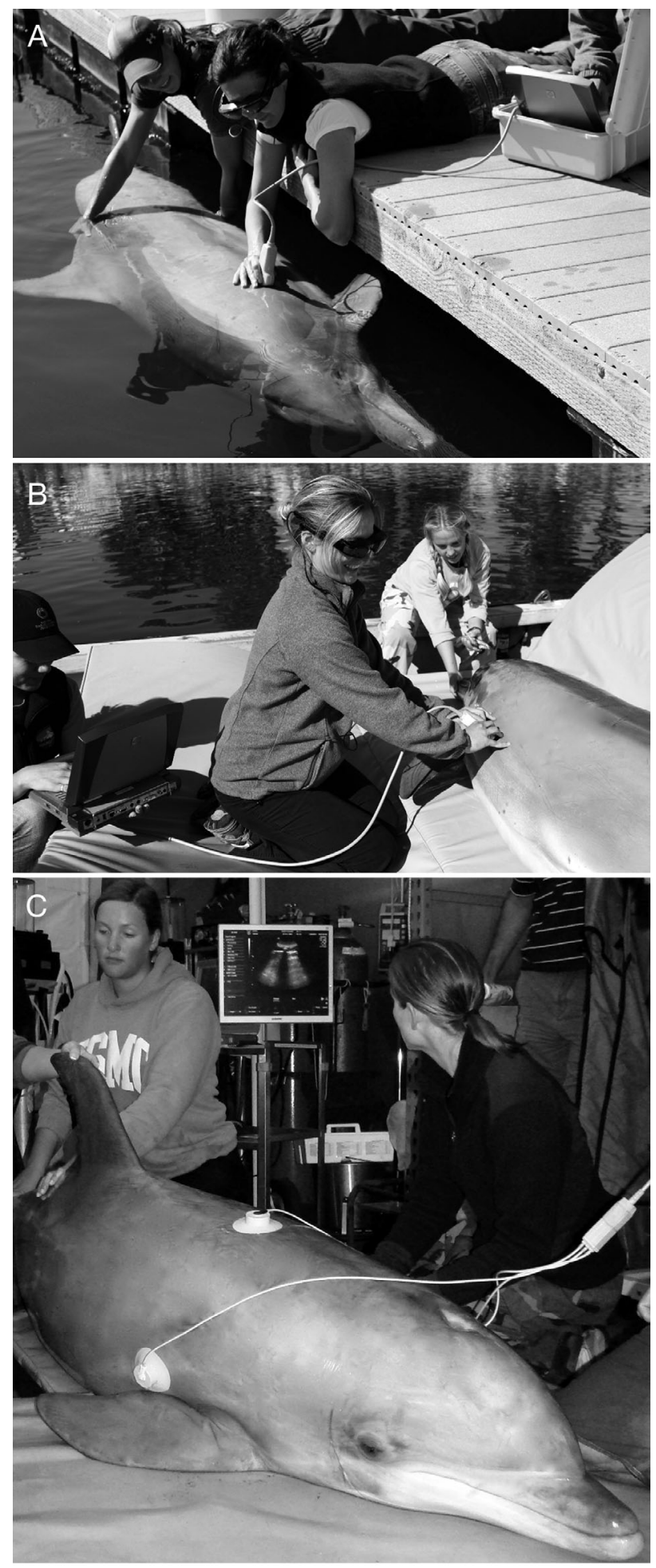

(Fig. 1A), (2) with the animal on land or in a boat adjacent to the enclosure (Fig. 1B), or (3) inside a veterinary hospital within close proximity to the ocean enclosure (Fig. 1C). When performed in the water, the dolphins were trained to voluntarily rest in lateral recumbency in the arms of the trainer, who stabilized the animal from the side of the enclosure or from a submerged stand. On land, exams were performed on foam pads and began with the animal in sternal recumbency. If medically stable, the animal was then rolled into lateral recumbency to allow for better visualization of the ventral portion of the lung fields. Dolphin skin is smooth and hairless, so no preparation of the skin was needed. When exams were performed in the water, ultrasound transmission gel was not needed as the water served as a coupling medium.

Two scanning techniques were used for evaluation. The first technique was previously described (Stone 1990, Brook et al. 2001), and began with the transducer in a dorsal plane (transducer perpendicular to the ribs) at the most craniodorsal aspect of the lung field. The sonographer then slid the probe caudally pausing at each intercostal space (the acoustic window) to fan dorsally and ventrally. When the most caudodorsal aspect of the lung field was reached, the sonographer dropped ventrally a short distance and repeated the technique, sliding caudal to cranial. For the second technique, the sonographer began at the craniodorsal point of the lung field, again with the probe in a dorsal plane but instead sliding ventrally in the intercostal space. At the most ventral aspect, the sonographer moved caudally to the next intercostal space and slid dorsally, repeating until the remainder of the lung field was scanned. When pleu-

Fig. 1. Tursiops truncatus. Ultrasound examination techniques. (A) Voluntary ultrasound exam. The dolphin floats on its side in an ocean enclosure supported by a trainer to facilitate the exam. The live ultrasound image is displayed in the video goggles worn by the sonographer, as the unit's LCD screen is not bright enough to overcome sunlight brightness. The sonographer communicates with the machine operator to optimize the image. (B) Ultrasound in a boat next to the ocean enclosure. The dolphin voluntarily slides out onto a foam pad and rests calmly during ultrasound examination. This technique also requires the use of video goggles and a technician operating the machine. (C) Ultrasound examination in the veterinary clinic. The dolphin either voluntarily slides out of the water onto a foam pad on a boat, or swims into a stretcher, and is then transported by crane or fork lift to the land-based clinic. Trainers keep the animals wet and comfortable while the veterinarian performs the exam. The sonographer can either directly operate the machine or have a technician operate the machine. The transducer (not seen) is placed in a similar fashion as in A and B 
ral and pulmonary abnormalities were identified, transverse and oblique planes were acquired to provide additional detail. The left and right marginal lymph nodes were detected at the mid-ventral point of each lung field, located in the pleural space caudolateral to the heart and against the diaphragm.

All exams were performed in real-time B-mode. Image settings varied according to the subject. As a general rule, the image brightness was decreased in the near field by decreasing the near gain and increased in the far field by increasing the far gain compensation function. The area of interest was placed in the mid-third of the screen by adjusting the depth. One focal zone was placed at the level of the region of interest when using the GE Voluson i. Tissue harmonic imaging was used when available, multi-beam was used on the Sonosite units, and speckle reduction imaging (low) was utilized with the GE Voluson i. The result was an image subjectively pleasing to the eye with the area of interest located in the middle of the screen. When appropriate, color Doppler was used to study blood flow patterns.

Dolphins were acclimated to being on land as part of their routine training and to facilitate their care. When out of the water, animals were kept wet, cool, and comfortable while resting on foam pads. Healthy animals rarely displayed significant changes in respiratory rate or character when on land. Placing ill animals on land was either minimized or avoided, as animals with respiratory compromise occasionally developed abnormal respiratory character, tachypnea, and/or dyspnea, particularly when significant lung disease was present. If warranted or further diagnostics were indicated, animals were transported to the land-based onsite veterinary hospital. All landbased examinations were designed for rapid and safe return of the dolphin to the water if needed.

\section{Fine needle aspirates and core biopsies}

When indicated, fine needle aspirates of pulmonary masses were performed by a previously described technique (Van Bonn \& Jensen 2001). The skin was cleaned with betadine and alcohol, followed by a local anesthetic block with $2 \%$ lidocaine from skin to intercostal muscle layer. The lidocaine was given 5 to $10 \mathrm{~min}$ to take effect, the area prepared again with betadine and alcohol, and a stab incision made with a no. 11 scalpel blade. A 3.5 inch $(\sim 8.9 \mathrm{~cm}), 18 \mathrm{~g}$ spinal needle attached to a standard bore 3-way stopcock with an extension set and a 10 to $20 \mathrm{~cm}^{3}$ syringe were used to aspirate the mass under ultrasound guidance. For cavitated masses that were difficult to aspirate, a small volume of sterile saline was infused to facilitate removal of material, which was most often purulent or inspissated material. Samples were processed for cytology, microbiological culture, and molecular diagnostics. Following the procedure, animals were monitored for complications for at least $30 \mathrm{~min}$, then rechecked 2 to $3 \mathrm{~h}$ later with ultrasound.

Core biopsies were collected when fine needle aspiration was not productive and/or lesions fit the following criteria: superficial, easy to access, limited blood supply, not fluid filled, and greater than $1 \mathrm{~cm}$ in diameter, to avoid accidental sampling of deeper tissues. Core biopsies were performed by a previously described technique (Lutmerding et al. 2010). Similar to the fine-needle aspirate technique, the skin was cleaned with betadine and alcohol, followed by a local anesthetic block with $2 \%$ lidocaine from skin to intercostal muscle layers. The lidocaine was given 5 to $10 \mathrm{~min}$ to take effect, the area prepared again with betadine and alcohol, and a stab incision made with a no. 11 scalpel blade. Using ultrasound guidance, a $6.8 \mathrm{~cm}, 17 \mathrm{~g}$ coaxial introducer needle was placed through the skin, blubber, and intercostal muscle layers, followed by a $10 \mathrm{~cm}$, $18 \mathrm{~g}$ BioPince full core biopsy instrument (Angiotech) that was advanced through the pleural lining and into the mass, carefully timing advancement of the instrument with respiration. Multiple biopsies were taken by leaving the introducer needle in place and entering the mass several times with the BioPince device, using slightly different angles for each biopsy. Core biopsies were processed for histopathology, culture, and molecular diagnostics. Animals were monitored for complications for at least $30 \mathrm{~min}$, then rechecked with ultrasound examination at 2 to $3 \mathrm{~h}$ post procedure.

\section{RESULTS}

From 2005 to 2010, a total of 498 non-cardiac thoracic ultrasound exams were performed on 79 bottlenose dolphins. We removed 28 exams from the study due to incomplete electronic records. The remaining 470 exam records were evaluated for the presence or absence of abnormal findings. The majority of exams had no abnormal pleural or pulmonary findings detected with ultrasound $(60.4 \%$ of the exams performed). In the remaining exams, abnormal sonographic findings were detected primarily during non- 
routine exams conducted to address clinical signs associated with illness or, more often, as follow-up exams to track previously identified lung abnormalities. For this reason, abnormal findings are overrepresented in the data set since lung abnormalities were typically tracked with repeat exams until resolution of disease. The following abnormalities were detected: pleural effusion (23.1\% of abnormal exams), pulmonary masses $(13.4 \%)$, alveolar-interstitial syndrome $(53.8 \%)$, and pulmonary consolidation $(9.7 \%)$.

\section{Normal lung}

In normal lung, the epidermis and hypodermis (blubber layer) were easily identified, as well as the underlying subcutaneous fat and muscular layers (Fig. 2). As expected, the ribs created acoustic shadows, and the intercostal muscles were seen between the ribs. Although individual muscular layers can be discerned, no effort was made to identify each inter-

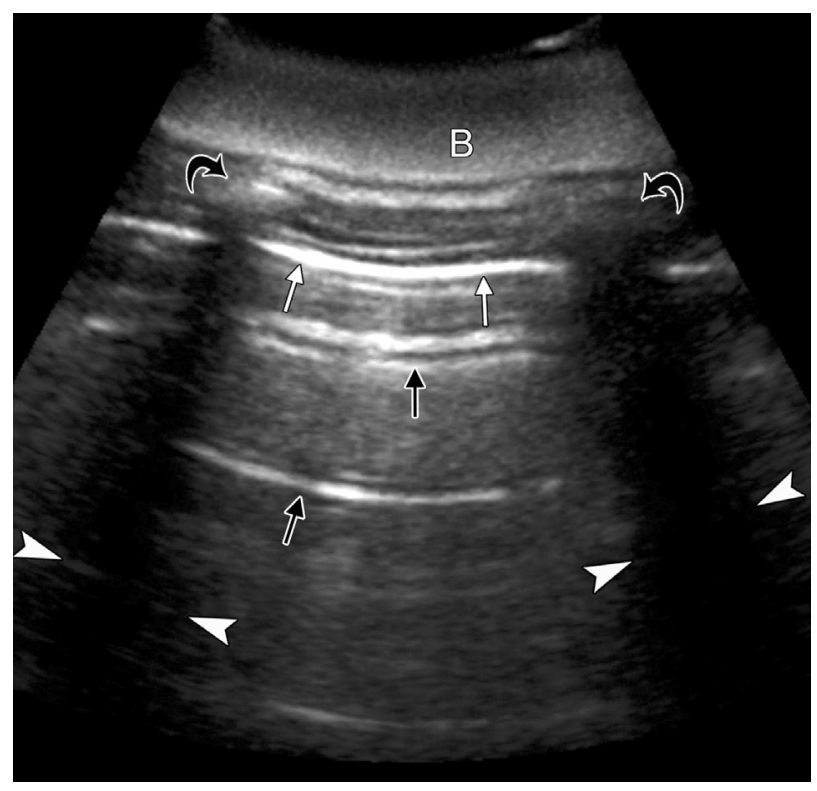

Fig. 2. Tursiops truncatus. Normal dorsal plane sonogram of the thorax of a 9 yr old female (cranial to the left) acquired with a 2 to $5 \mathrm{MHz}$ curvilinear transducer. The thick hypodermis or blubber layer (B) is diffusely echogenic and is located in the near field lateral to the ribs (curved arrows). Acoustic shadowing (arrowheads) from the ribs and multiple reverberation artifacts (black arrows) are noted. The latter are created by the visceral pleural intimately associated with the most superficial portion of the lung (white arrows). Due to air in the lungs and the presence of the reverberation artifacts, normal lung parenchyma cannot be imaged with ultrasound. The echogenic tissue between the blubber and the surface of the lung represents the intercostal musculature costal muscle. The pleural line, created by the visceral pleura against air-filled lung, was seen as a hyperechoic horizontal line in the intercostal space below the intercostal muscle layer. The parietal pleura was difficult to distinguish in the healthy animal; however, sliding of the lung at the level of the interface between the parietal and visceral pleura could be seen during breath cycles. A reverberation artifact was created by the tissue-air interface of the visceral pleura and normally air-filled lung, which was seen as multiple lines below the pleural line.

A few comet-tail artifacts, defined as short or small hyperechoic lines that tapered in width and decreased in echogenicity with depth (Lichtenstein et al. 1997, Agricola et al. 2005, Louvet \& Bourgeois 2008), were occasionally found as the only thoracic ultrasound finding in an otherwise healthy animal. Although not properly characterized at the time, sporadic ringdown artifacts, defined as hyperechoic beams that originated at the pleural line and extended to the bottom of the image without decreasing in intensity (Louvet \& Bourgeois 2008), were also seen in otherwise healthy animals. These artifacts were typically seen most often in the caudoventral lung fields and in the ventral lung field surrounding the heart where the lung tissue becomes very thin. Comet-tail and ring-down artifacts are generally nonspecific and can be generated by both normal and abnormal structures (Sartori \& Tombesi 2010) and in healthy patients (Louvet \& Bourgeois 2008). The artifact in itself does not represent a structure, but rather the way the sound interacts with the tissue in question. However, if either comet-tail or ring-down artifacts were found diffusely throughout the lung fields, in clusters, or associated with other abnormalities, they were considered abnormal.

\section{Pleural effusion}

Pleural effusion was readily detected between the parietal and visceral pleura. When feasible, thoracocentesis was performed under ultrasound guidance to obtain fluid samples (Fig. 3). Pleural effusion varied from anechoic (cases with transudate and modified transudates) to mildly echogenic (one case of hemothorax). Occasionally, a small amount of anechoic fluid was seen cranial to the diaphragm at the mid-ventral aspect of the lung field close to the cardiac window and marginal lymph node. When fluid could not be appreciated elsewhere in the lung field and no significant clinical signs were present, the fluid was considered within normal limits. 


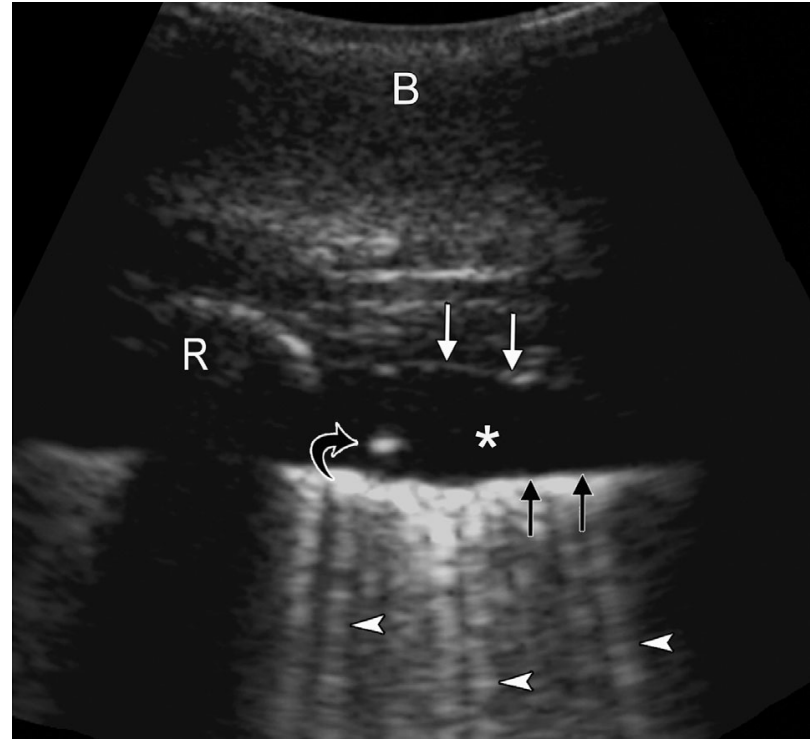

Fig. 3. Tursiops truncatus. Dorsal plane sonogram of the thorax of a $45 \mathrm{yr}$ old male taken with a 2 to $5 \mathrm{MHz}$ curvilinear transducer during thoracocentesis to obtain a sample of pleural fluid (cranial to the left). There is a mild to moderate volume of pleural effusion ( $*$ ) between the visceral (black arrows) and parietal pleura (white arrows). The free fluid is surrounding the tip of the needle (curved arrow) used to perform thoracocentesis. Multiple comet-tail and ring-down artifacts (arrowheads) are obscuring portions of the lung parenchyma. The animal responded to diuretic therapy, resulting in improvement or resolution of the pleural effusion. Gross necropsy revealed cardiomegaly, and histopathology confirmed cardiomyopathy, secondary hepatic congestion, and severe pulmonary granulomatous inflammation and necrosis due to a systemic Cryptococcus neoformans infection. The interface of a rib $(\mathrm{R})$ is noted cranial and lateral to the tip of the needle. The layer of blubber (B) can be seen in the near field

\section{Pulmonary masses}

Pulmonary masses were detected on the periphery of the lungs. Five dolphins with masses were sampled by fine-needle aspiration, core biopsy, or at necropsy. Of these, 2 were diagnosed with fungal abscesses and 3 with bacterial abscesses. The abscesses were typically hypoechoic but some contained anechoic centers, hyperechoic foci, or patterns of mixed echogenicity (Figs. 4 \& 5). Iatrogenic hemothorax developed in one case during biopsy of a fungal abscess, and the clinician determined that it was possible that an intercostal artery was damaged during introducer needle placement. The animal had no outward clinical signs, and the condition resolved with thoracocentesis and rest. Mild, localized iatrogenic pneumothorax was occasionally detected immediately following needle or instru-

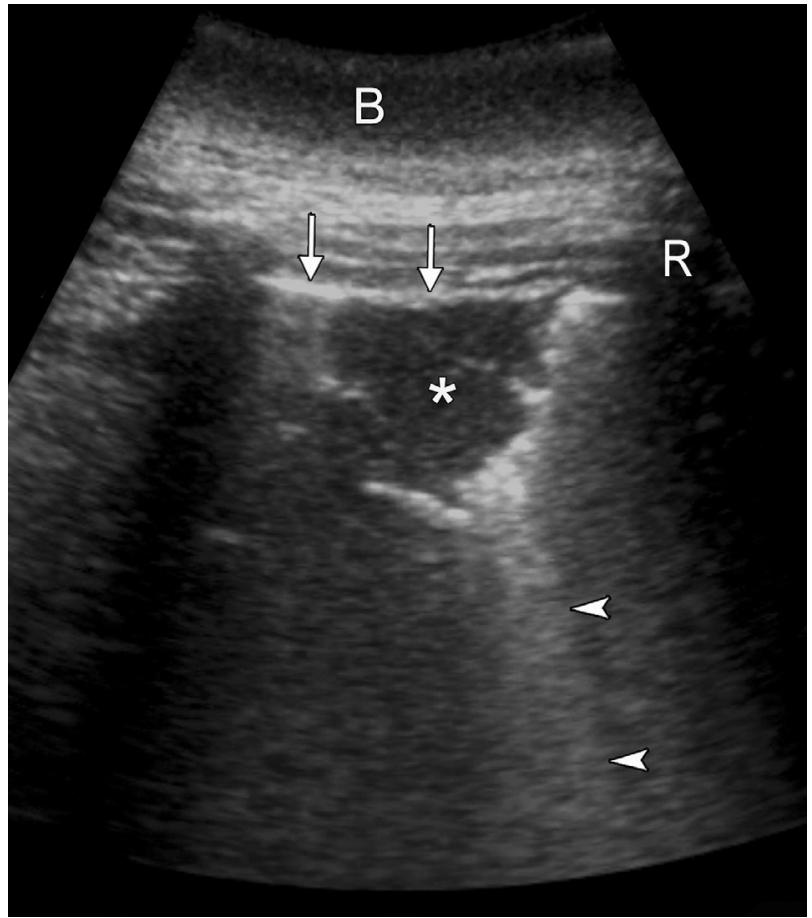

Fig. 4. Tursiops truncatus. Dorsal plane sonogram of the thorax of a 26 yr old female taken with a 2 to $5 \mathrm{MHz}$ curvilinear transducer of a pulmonary mass in the right caudal lung lobe (cranial to the left). There is a homogenously hypoechoic parenchymal mass with irregular borders (*). The lesion lacks an extra-pleural sign and preserves the immediately adjacent parietal pleura (white arrows), indicating that the lesion is of pulmonary origin. Reverberation artifacts generated by the mass/lung parenchyma interface can also be seen (arrowheads). Ultrasound-guided fine needle aspirate and CT-guided core biopsies confirmed that the mass was a Coccidiodes immitus abscess. The layer of blubber (B) and the interface of a rib (R) are also seen

ment removal, but with no associated clinical signs. This was due to a small amount of free air in the pleural space, resulting in either a partial or complete loss of visualization of the region of interest, and resolved without intervention.

\section{Alveolar-interstitial syndrome}

Alveolar-interstitial syndrome (AIS) was defined as an increase of fluid or cellular infiltrate in the interstitium and reduced air in the alveolar spaces, with or without interlobular septal thickening (Volpicelli et al. 2006, Louvet \& Bourgeois 2008, Cardinale et al. 2009). The syndrome is characterized by the presence of multiple comet-tail and/or ring-down artifacts throughout the lung fields, often clustered and seen in higher frequency than ex- 


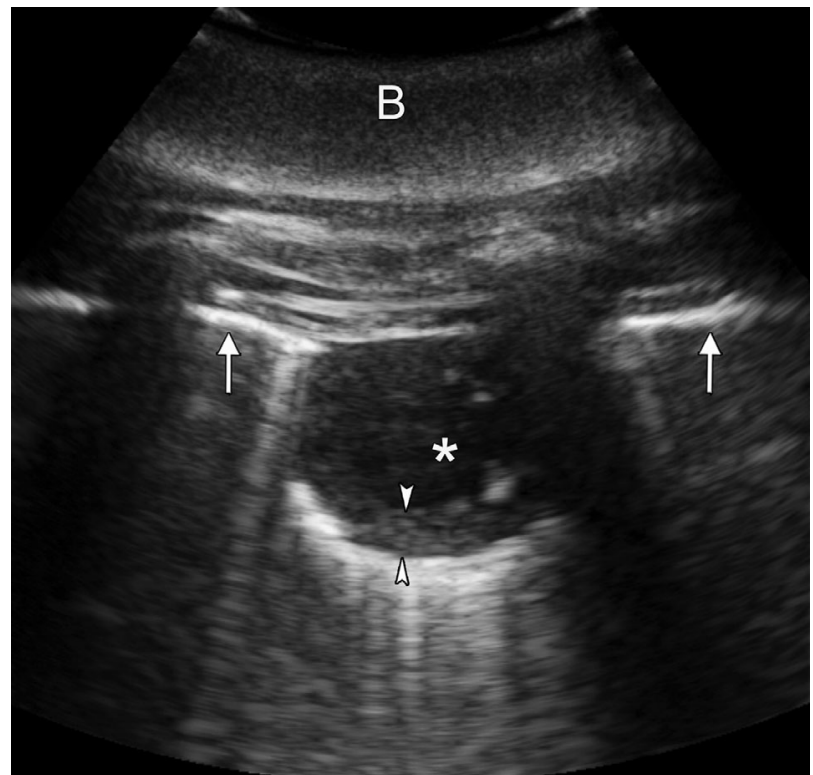

Fig. 5. Tursiops truncatus. Dorsal plane sonogram of the thorax of a $28 \mathrm{yr}$ old female taken with a 2 to $5 \mathrm{MHz} 3 \mathrm{D} / 4 \mathrm{D}$ curvilinear transducer of a pulmonary bacterial abscess (cranial to the left). The lesion has a hypoechoic center ( $*$ ), a concentric echogenic outer layer (arrowheads), and a few hyperechoic foci likely representative of necrotic tissue. The mass abuts the parietal pleura (white arrows). Ultrasoundguided fine needle aspiration with cytology, culture, and bacterial PCR resulted in a diagnosis of Brucella maris pulmonary abscess. The layer of blubber (B) is seen in the near field

pected (refer to normal lung description above). AIS includes disease states that are either acute (pulmonary edema, acute respiratory distress, interstitial pneumonia) or chronic (pulmonary fibrosis, chronic interstitial lung diseases; Volpicelli et al. 2006, Louvet \& Bourgeois 2008, Gargani 2011, Stefanidis et al. 2011). Concurrent evidence of pleural effusion may or may not be seen with the syndrome. As stated previously, comet-tail and ring-down artifacts can be generated by both normal and abnormal structures (Sartori \& Tombesi 2010). While the artifact can be seen as the result of the highly reflective nature of the normal pleura, the artifacts were subjectively increased in number and frequency in cases of interstitial pneumonia (Fig. 6), cardiogenic (Fig. 3) and noncardiogenic pulmonary edema, and pulmonary fibrosis (Fig. 7). This is consistent with findings reported in both the human and veterinary literature (Volpicelli et al. 2006, Louvet \& Bourgeois 2008, Cardinale et al. 2009, Barillari \& Fioretti 2010, Sartori \& Tombesi 2010, Gargani 2011, Stefanidis et al. 2011).

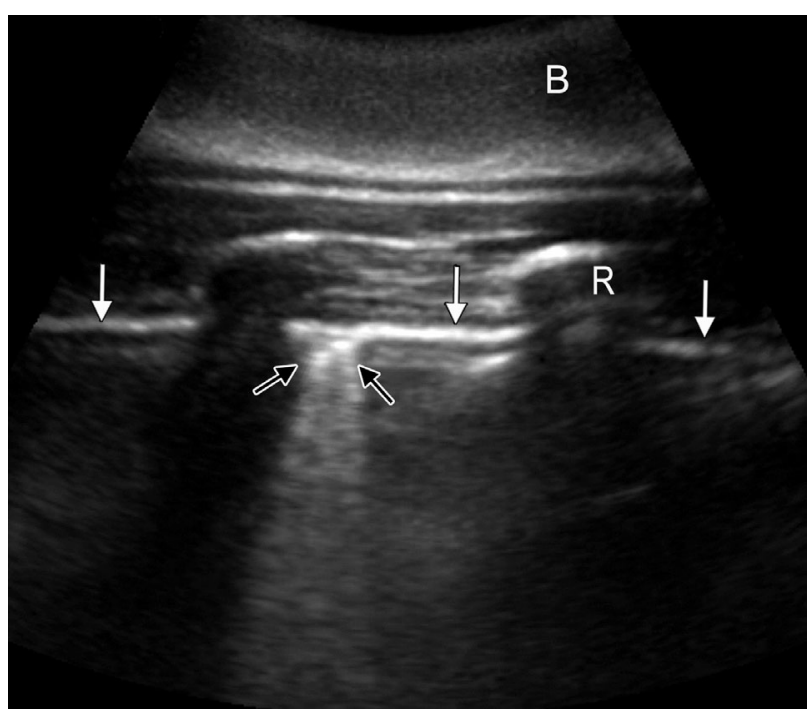

Fig. 6. Tursiops truncatus. Dorsal plane sonogram of the thorax of a 40 yr old male taken with a 2 to $5 \mathrm{MHz}$ curvilinear transducer (cranial to the left). There is a loss of reverberation artifact (cf. Fig. 2) and a small cluster of ring-down artifacts (black arrows), which were found throughout the lung fields but predominantly in the cranial and mid-lung lobes. The visceral pleura (white arrows) is smooth and well-defined throughout with the exception of the area where the ring-down artifact is generated. The layer of blubber (B) and the interface of a rib (R) are also seen. Histopathology confirmed a diagnosis of pneumonia, and bacterial cultures of the lung were positive for Streptococcus zooepidemicus

\section{Pulmonary consolidation}

AIS can progress to fluid and/or infiltrate accumulation in the alveolar spaces, resulting in pulmonary consolidation (Fig. 8). Consolidation was defined as fluid or infiltrate accumulation in the alveoli, often resulting in hepatization (Stowater \& Lamb 1989, Targhetta et al. 1992, Jung \& Bostedt 2004, Larson 2009), or a liver-like appearance to the lung. The affected portion of the lung resembles the ultrasonographic appearance of the liver, similar to that in other species. The term hepatization has been used to describe such appearance even though the term originates from the appearance of diseased lung as seen in histopathological slides. Trapped air within the airways, or sonographic air bronchograms, were detected as multiple, strongly hyperechoic linear interfaces and punctate foci generating reverberation artifacts. Fluid bronchograms were also seen. The fluid bronchogram is a fluid-filled bronchus which is seen as a tubular structure with anechoic luminal contents, differentiated from vasculature with color 


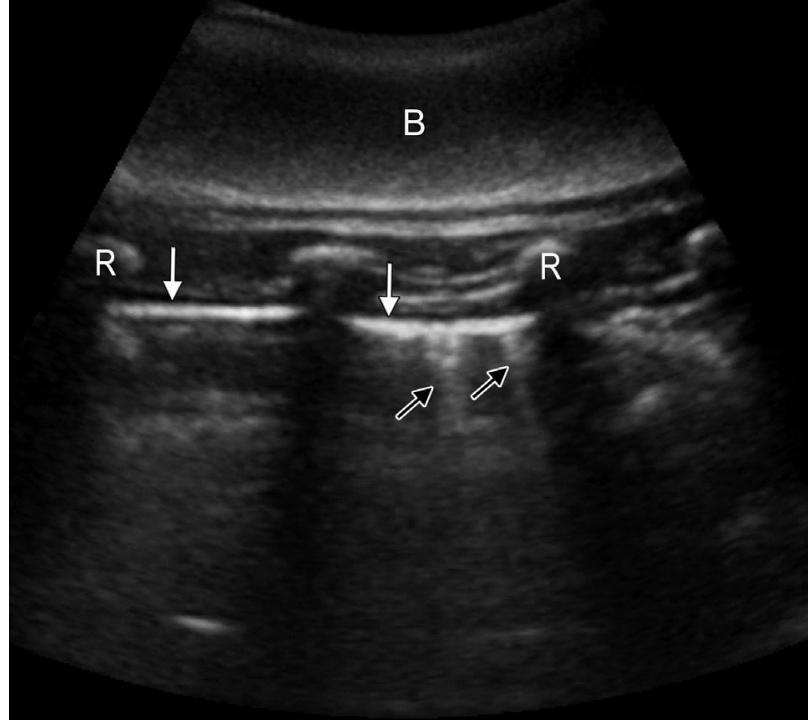

Fig. 7. Tursiops truncatus. Dorsal plane sonogram of the thorax of a $30 \mathrm{yr}$ old male taken with a 2 to $5 \mathrm{MHz}$ curvilinear transducer (cranial to the left). A few comet-tail artifacts are seen (black arrows), suggestive of alveolar-interstitial disease. The visceral pleura is smooth (white arrows). Minor irregularities in the pleura are seen at the level where the comet-tail artifacts are generated. The blubber (B) and the interfaces of several ribs (R) are also seen. A presumptive diagnosis of fibrosis was made based on thoracic radiographs and clinical signs

flow interrogation. The fluid bronchogram is highlighted by the surrounding pulmonary consolidation (Goerg 2006). The 4 animals in which consolidation was seen ultrasonographically were also diagnosed with pneumonia, atelectasis, and/or pulmonary edema on the basis of clinical presentation, cytology, or histopathology. Specifically, 2 geriatric animals were diagnosed with combined consolidation and atelectasis secondary to pleural effusion and pulmonary edema; and 2 adult animals with fungal pneumonia had evidence of pulmonary consolidation.

\section{Marginal lymph nodes}

During the study period, marginal lymph nodes were detected but not specifically evaluated by the sonographer unless obvious pathology was present, characterized by gross enlargement or a change in echogenicity. The marginal lymph node has been previously reported to measure $5 \times 2 \times 2 \mathrm{~cm}$ on post mortem exam (Cowan \& Smith 1999). For the purpose of these ultrasound exams, we were conservative in evaluating node enlargement. In general, if the marginal lymph node measured greater than

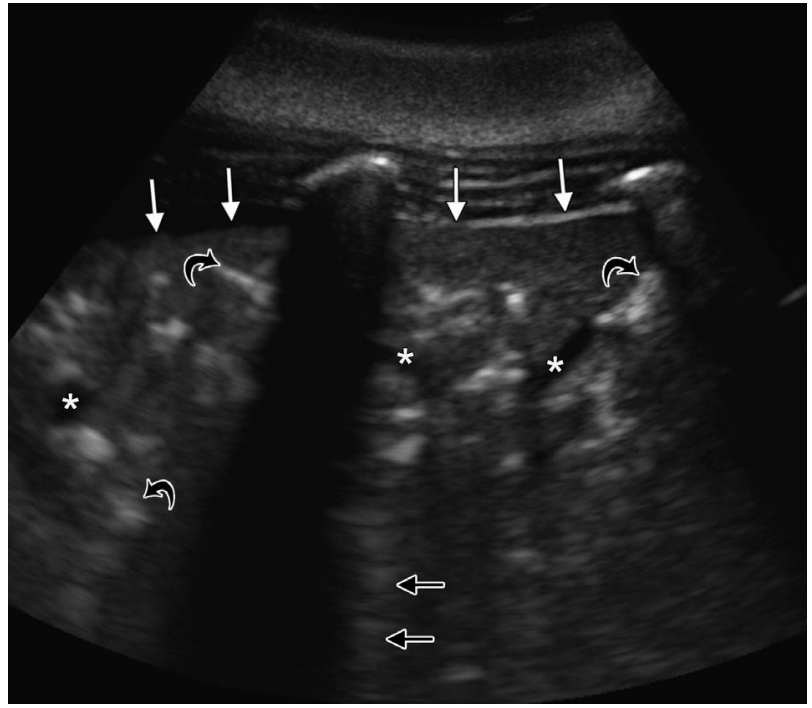

Fig. 8. Tursiops truncatus. Dorsal plane sonogram of the thorax of a $51 \mathrm{yr}$ old female taken with a 2 to $5 \mathrm{MHz} 3 \mathrm{D} / 4 \mathrm{D}$ curvilinear transducer (cranial to the left). Diffuse pulmonary consolidation is noted. The ubiquitous reverberation artifacts, generated by normally aerated lung parenchyma, are no longer present (cf. Fig. 2). Instead, a homogenously echogenic parenchyma is seen, resembling the ultrasonographic appearance of the liver and referred to as hepatization. The surface of the lung borders is clearly visible (white arrows). Overlying the heads of the first 3 straight white arrows, the lung is separate from the costal wall by a small amount of anechoic free fluid. Scattered hyperechoic lines and foci (curved arrows) are also seen, indicative of partially aerated lung tissue. The tubular anechoic structures $(*)$ represent either pulmonary vasculature of fluid-filled bronchi; Doppler ultrasound is needed to differentiate the two. Faintly defined reverberation artifact is generated from the foci of gas present in the affected lung parenchyma (black arrows). The animal was diagnosed with right-sided dilated cardiomyopathy by echocardiography, which was confirmed at necropsy

$5 \mathrm{~cm}$ in any plane, it was considered enlarged. In animals with no abnormal sonographic findings in the lungs and no evidence of respiratory disease, lymph nodes appeared relatively homogenous with smooth borders (Fig. 9).

\section{DISCUSSION}

Thoracic ultrasound examination of dolphins was easily and rapidly performed following the techniques described in this manuscript and provided valuable diagnostic information for the detection, tracking, and management of pleural and pulmonary diseases. Dolphin thoracic exams yielded sonographic findings similar to those of other animals and humans. In the majority of routine exams, no abnor- 


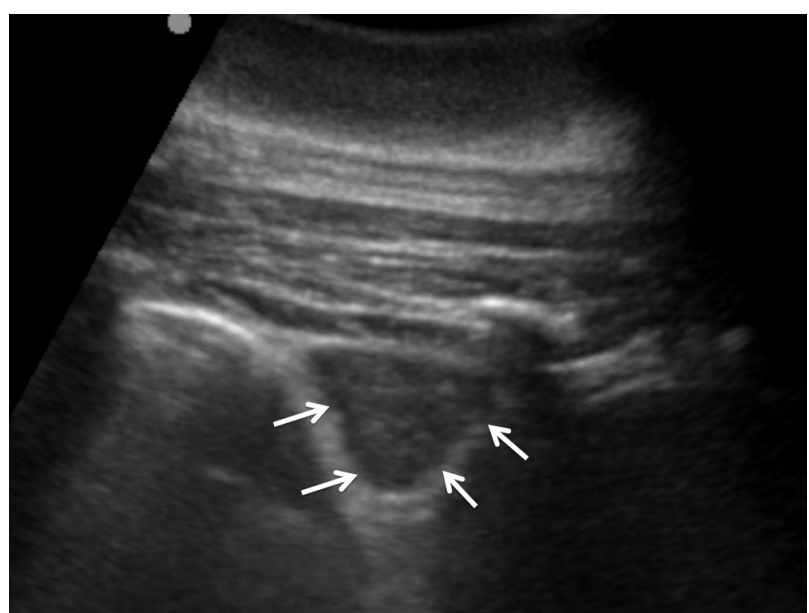

Fig. 9. Tursiops truncatus. Dorsal plane sonogram of the left marginal lymph node of a clinically normal 27 yr old male taken with a 2 to $5 \mathrm{MHz}$ curvilinear transducer (cranial to the left). The node (white arrows) has a relatively homogenous echogenicity and smooth borders

mal pleural or pulmonary findings were detected with ultrasound. The most common abnormal findings in order of prevalence were AIS, pleural effusion, pulmonary masses, and pulmonary consolidation. These findings were typically detected during non-routine exams to identify and track disease progression or resolution, and therefore abnormal results are overrepresented. AIS was generally nonspecific as it represented several possible disease states, ranging from mild to severe, acute to chronic, and active to inactive conditions. The diagnosis of pleural effusion, pulmonary masses, and pulmonary consolidation was more straightforward to assess clinical significance. As expected, pairing ultrasound findings with clinical signs was essential to determine relevance.

Both scanning techniques, sliding cranial-to-caudal and sliding dorsal-to-ventral, provided valuable data. However, we preferred the dorsal-to-ventral technique, as it was subjectively more thorough. By sliding the probe dorsal to ventral between 2 ribs and then repeating in the next rib space ventral to dorsal, the ultrasonographer was less likely to overlook an area of the lung. When scanning cranial to caudal, the sonographer could drift from the intended dorsal plane, missing a portion of the lung in the process. The dorsal-to-ventral technique also eliminated the need to fan dorsally and ventrally, which could reduce artifacts created by scanning in oblique planes.

Due to the high survivability of the US Navy dolphin population (Venn-Watson et al. 2011a) and suc- cessful treatment outcomes, gross pathology and histopathology data were often unavailable for definitive confirmation of ultrasound findings. Fine needle aspirates and lung biopsies were utilized in specific cases to obtain additional data and often yielded definitive diagnoses. Percutaneous sampling of pulmonary masses is recommended when sampling is considered low risk to the patient, for example, when masses are superficial, easy to access, and have limited blood supply. The blood supply to pulmonary masses was based on subjective interrogation of the lesions with color Doppler. The risks include iatrogenic pneumothorax or hemothorax, lung tissue trauma, and contamination of the lungs if improper techniques are used.

Spontaneous pneumothorax is a rarely diagnosed condition in dolphins and when present can significantly impact buoyancy. During the study period, no cases of spontaneous pneumothorax were detected with ultrasound, and no dolphins displayed outward clinical signs consistent with this condition. Mild iatrogenic pneumothorax was seen secondary to percutaneous sampling of pulmonary masses, which resolved without therapy.

Pleural effusion was readily diagnosed with ultrasound and was detected in $23 \%$ of the abnormal thoracic sonograms. Two geriatric dolphins had severe effusions secondary to congestive heart failure, one adult dolphin had a confirmed hemothorax, and the remainder had smaller volumes that were readily seen cranial to the diaphragm but were difficult to detect throughout the pleural cavity. Similar findings of mild effusions were seen in a previous epizootic of parainfluenza within the US Navy population (VennWatson et al. 2008), and these pleural effusions were occasionally accompanied by pericardial and/or peritoneal effusions. Based on these data, infectious pneumonias are considered a potential cause of lowgrade pleural effusion in dolphins. Small volumes of fluid were often found in the mid-ventral portion of the pleural cavity in otherwise healthy animals, specifically in the acoustic window where the apex of the heart and diaphragm were both seen. If no other fluid could be found in the pleural cavity, the fluid was considered within normal limits.

Peripheral pulmonary masses that abutted the pleural line were readily identified with ultrasound. Lesions greater than $1 \mathrm{~cm}$ in diameter were typically sampled with ultrasound-guided fine needle aspiration and/or core biopsy for further evaluation. The $1 \mathrm{~cm}$ minimum diameter guideline was based on the assumption that this would decrease the risk of accidental sampling of deeper tissues. Half of the lesions 
detected were either bacterial or fungal in origin, as determined by microbiological culture and/or molecular diagnostics. The etiology of the other lesions remained unknown. Neoplasia was considered unlikely, as only one bottlenose dolphin case of pulmonary neoplasia has been reported in the scientific literature (Ewing \& Mignucci-Giannoni 2003). There have been no documented cases of pulmonary neoplasia in this dolphin population in over $30 \mathrm{yr}$ (VennWatson et al. 2012).

In one of the pulmonary mass cases, a well-circumscribed round peripheral lesion was detected with ultrasound, immediately sampled, and bacterial culture results were positive for Staphylococcus aureus. Necropsy revealed multiple deep abscesses that could not be seen with ultrasound. In 2 other cases where peripheral lesions were found with ultrasound and confirmed to be infectious, computed tomography (CT) exams were performed and revealed additional lung lesions. Although our sample size was small, we would advise clinicians to be wary of solitary lesions if an infectious process is suspected or confirmed, and consider the likelihood of multiple lesions being present. As with companion animals, thoracic radiographs or CT exams are better suited than ultrasound to assess the entirety of the lung parenchyma.

In-house epidemiologic analysis of $30 \mathrm{yr}$ of lung histopathology data at the US Navy showed that the most common lung abnormality was pneumonia, with $71.8 \%$ of pneumonia cases having either a bacterial or fungal etiology (Venn-Watson et al. 2012). Pneumonia has been commonly reported in stranded wild dolphins (Diamond et al. 1979, Di Guardo et al. 1995, Bogomolni et al. 2010), and unlike the US Navy dolphins, many of these pneumonias involved parasitism. The US Navy population of dolphins has been routinely dewormed as part of their preventive medicine program, and there have been no reports of verminous pneumonia found at necropsy in the past $30 \mathrm{yr}$. Therefore, this ultrasound study was likely limited to cases of pneumonia with bacterial and fungal etiologies, as well as suspect viral pneumonias based on case presentation and antibody seroconversion data (Venn-Watson et al. 2008).

Reverberation artifacts also known as comet-tail artifacts (Lichtenstein et al. 1997, Agricola et al. 2005, Louvet \& Bourgeois 2008) were identified as abnormal in frequency and/or distribution in just over half of the abnormal exams. These artifacts were considered nonspecific indicators of alveolar-interstitial diseases and/or pulmonary consolidation. As mentioned, we incorrectly characterized ring-down arti- facts as comet-tail artifacts throughout the study period. We are not able to differentiate the prevalence of the 2 artifacts for the purpose of this study. Instead, we assumed that findings of reverberation (comet-tail) artifacts represented comet-tails only, ring-downs only, or a combination of the 2 artifacts. The clinical significance of appropriately differentiating between these 2 artifacts is unknown. We should note that although increased frequency and broad distribution of artifacts were determined to be important indicators of pulmonary disease, which is consistent with the human literature (Avruch \& Cooperberg 1985, Lichtenstein et al. 1997, Agricola et al. 2005, Louvet \& Bourgeois 2008, Sartori \& Tombesi 2010), we cannot definitely state the source of the artifacts without histological data.

Ring-down artifacts have been documented in cases of pleuritis in humans and animals (Avruch \& Cooperberg 1985, Louvet \& Bourgeois 2008). Although we are not specifically reporting on pleuritis, pleural roughening or thickening was reported occasionally in the ultrasound records. Histopathology data demonstrated that pleuritis was found in $10.7 \%$ of all abnormal lung tissue from Navy dolphins between 1980 and 2010 (Venn-Watson et al. 2012). Therefore, there is a need to standardize pleural thickness evaluation, which would involve consistently measuring pleural thickness, developing a normal range, and documenting irregularities, roughening, and changes in thickness. The low frequency probes used for this study may not be appropriate for accurately evaluating the pleura. Higher frequency probes should be evaluated for this purpose prior to standardization of the technique.

Pulmonary fibrosis was arguably the most challenging diagnosis to make based on sonographic findings. In the historical US Navy histopathology data set, pulmonary fibrosis was the second most common finding (Venn-Watson et al. 2012). However, only one dolphin was suspected to have pulmonary fibrosis based on a history of tachypnea when out of the water and abnormal radiographic findings. In humans, pulmonary fibrosis is associated with evidence of AIS (Volpicelli et al. 2006, Gargani 2011, Stefanidis et al. 2011), characterized by comettail and/or ring-down artifacts. Although we documented an increased frequency and clustering of these artifacts in this case, we were not able to definitely diagnose fibrosis due to the nonspecific nature of the sonographic findings and lack of sampling to obtain a histological diagnosis. Dolphins under human care often live beyond $40 \mathrm{yr}$ of age, warranting the need for geriatric medicine programs (Venn- 
Watson et al. 2011a,b). As a disease associated with aging, pulmonary fibrosis will be increasingly important to understand and diagnose. Future efforts should focus on comparing ultrasound, radiograph, and CT imaging modalities with pulmonary function testing to identify the best methods of detection.

Pulmonary consolidation was rarely detected. Two cases of combined atelectasis and consolidation secondary to pleural effusion and pulmonary edema were detected in geriatric animals, and 2 cases of severe fungal pneumonia resulted in consolidation. Hepatization of the lungs was readily apparent in the cases of combined atelectasis and consolidation. Fungal pneumonia was characterized by focal consolidation of the lungs, supported by radiographic and CT findings. Cases of confirmed bacterial and suspected viral pneumonia did not have associated sonographic findings of consolidation in the present study; however, consolidation has commonly been reported in both bacterial and viral pneumonias in the human and veterinary literature. Pulmonary consolidation has also been associated with neoplasia, lung torsion, and contusions (Gargani 2011, Stefanidis et al. 2011), but none of these conditions was suspected in this study based on historical histopathological findings or clinical signs of the animals examined.

The left and right marginal lymph nodes, which drain the lung lobes (Cowan \& Smith 1999), were not consistently evaluated throughout the study period. However, these nodes were specifically included in lung ultrasound examinations beginning in 2009. They were detected at the mid-ventral point of each lung field, caudal to the cardiac window and cranial to the diaphragm. The external landmark for this lymph node was just caudoventral to the insertion of the pectoral flipper. We used conservative criteria to evaluate enlargement and echogenicity. We expect these criteria to become more refined as data are collected on the normal size range and echo pattern when examined with ultrasound.

One limitation of this study was the number of clinicians with various skill levels performing the ultrasound exams. This limitation was minimized by training, mentorship, and oversight by senior clinicians. When significant abnormalities were detected, senior clinicians would either attend or perform an exam to confirm findings. These practices allowed for standardization of techniques and interpretation.

As with all species, ultrasound was only capable of detecting diseases that involved the peripheral lung fields. It is important to emphasize that a major limitation of lung ultrasound is that lesions deep within the aerated lung or obscured by the ribs will be missed. This includes lesions only a few centimeters deep to the visceral pleura of the lung. Hence, a normal ultrasound of the lung does not rule out pulmonary disease. Additional imaging modalities such as radiographs and $\mathrm{CT}$ remain essential for detecting deep lung disease and establishing severity. Additional studies are needed to compare ultrasound, radiograph, and $\mathrm{CT}$ findings in the detection of specific pleural and pulmonary diseases in the bottlenose dolphin.

The number of thoracic ultrasounds performed within the 5 yr study period (498) highlights the increasing importance of ultrasound in marine mammal medicine. Ultrasound accurately and rapidly detected several pleural and pulmonary abnormalities in bottlenose dolphins, and when ultrasoundguided procedures enabled additional sampling for cytology, histopathology, culture, and/or molecular diagnostics, definitive diagnoses were often made.

Acknowledgements. We thank the Office of Naval Research for funding support of this project (award no. N0001411WX2 0241). We also thank the veterinarians who contributed to this paper through case management and ultrasound examinations, particularly W. Van Bonn and C. Dold. V. Cendejas provided invaluable technical support to the clinical veterinarians. The Navy Marine Mammal Program biotechnicians, trainers, and managers were instrumental in facilitating exams. J. Behm assisted in the literature review. Special thanks to S. Ridgway and S. Venn-Watson for their review of this manuscript.

\section{LITERATURE CITED}

Agricola E, Bobe T, Oppizzi M, Marino G, Zangrillo A, Margonato A, Picano E (2005) 'Ultrasound comet-tail images': a marker of pulmonary edema: a comparative study with wedge pressure and extravascular lung water. Chest 127:1690-1695

> Avruch L, Cooperberg PL (1985) The ring-down artifact. J Ultrasound Med 4:21-28

> Baker JR (1992) Causes of mortality and parasites and incidental lesions in dolphins and whales from British waters. Vet Rec 130:569-572

> Barillari A, Fioretti M (2010) Lung ultrasound: a new tool for the emergency physician. Intern Emerg Med 5:335-340

> Bogomolni AL, Pugliares KR, Sharp SM, Patchett K and others (2010) Mortality trends of stranded marine mammals on Cape Cod and southeastern Massachusetts, USA, 2000 to 2006. Dis Aquat Org 88:143-155

Bonar CJ, Boede EO, Garcia Hartmann M, LowensteinWhaley J and others (2007) A retrospective study of pathological findings in the Amazon and Orinoco river dolphin (Inia geoffrensis) in captivity. J Zoo Wildl Med 38:177-191

Brook F, Van Bonn W, Jensen E (2001) Ultrasonography. In: Dierauf LA, Gulland FMD (eds) CRC handbook of mar- 
ine mammal medicine, 2nd edn. CRC Press, Boca Raton, FL, p 593-620

> Cardinale L, Volpicelli G, Binello F, Garofalo G, Priola SM, Veltri A, Fava C (2009) Clinical application of lung ultrasound in patients with acute dyspnea: differential diagnosis between cardiogenic and pulmonary cause. Radiol Med (Torino) 114:1053-1064

> Cardinale L, Ardissone F, Garetto I, Marci V, Volpicelli G, Solitro F, Fava C (2010) Imaging of a benign solitary fibrous tumor of the pleura: a pictorial essay. Rare Tumors 2:e1

Carroll JM, Jasmin AM, Baucom JN (1968) Pulmonary aspergillosis of the bottlenose dolphin (Tursiops truncatus). Am J Vet Clin Pathol 2:139-140

> Cornaglia E, Rebora L, Gilli C, Di Guardo G (2000) Histopathological and immunohistochemical studies on cetaceans found stranded on the coast of Italy between 1990 and 1997. J Vet Med A Physiol Pathol Clin Med 47: 129-142

> Cowan DF, Smith TL (1999) Morphology of the lymphoid organs of the bottlenose dolphin, Tursiops truncatus. J Anat 194:505-517

> Cusick PK, Bullock BC (1973) Ulcerative dermatitis and pneumonia associated with Aeromonas hydrophilia infection in the bottle-nosed dolphin. J Am Vet Med Assoc 163:578-579

> Dailey M, Walsh M, Odell D, Campbell T (1991) Evidence of prenatal infection in the bottlenose dolphin (Tursiops truncatus) with the lungworm Halocercus lagenorhynchi (Nematoda: Pseudaliidae). J Wildl Dis 27:164-165

> Di Guardo G, Agrimi U, Morelli L, Cardeti G, Terracciano G, Kennedy S (1995) Post mortem investigations on cetaceans found stranded on the coasts of Italy between 1990 and 1993. Vet Rec 136:439-442

Di Guardo G, Marruchella G, Agrimi U, Kennedy S (2005) Morbillivirus infections in aquatic mammals: a brief overview. J Vet Med A Physiol Pathol Clin Med 52:88-93

$>$ Diamond SS, Ewing DE, Cadwell GA (1979) Fatal bronchopneumonia and dermatitis caused by Pseudomonas aeruginosa in an Atlantic bottle-nosed dolphin. J Am Vet Med Assoc 175:984-987

Domingo M, Visa J, Pumarola M, Marco AJ, Ferrer L, Rabanal R, Kennedy S (1992) Pathologic and immunocytochemical studies of morbillivirus infection in striped dolphins (Stenella coeruleoalba). Vet Pathol 29:1-10

Dunn JL, Buck JD, Robeck TR (2001) Bacterial diseases in cetaceans and pinnipeds. In: Dierauf LA, Gulland FMD (eds) CRC handbook of marine mammal medicine, 2nd edn. CRC Press, Boca Raton, FL, p 309-35

Elad D, Morick D, David D, Scheinin A, Yamin G, Blum S, Goffman O (2011) Pulmonary fungal infection caused by Neoscytalidium dimidiatum in a Risso's dolphin (Grampus griseus). Med Mycol 49:424-426

Ewing RY, Mignucci-Giannoni AA (2003) A poorly differentiated pulmonary squamous cell carcinoma in a freeranging Atlantic bottlenose dolphin (Tursiops truncatus). J Vet Diagn Invest 15:162-165

Fauquier DA, Kinsel MJ, Dailey MD, Sutton GE, Stolen MK, Wells RS, Gulland FMD (2009) Prevalence and pathology of lungworm infection in bottlenose dolphins Tursiops truncatus from southwest Florida. Dis Aquat Org 88: 85-90

Fiebiger J (1916) On structural peculiarities in the dolphin lung and their physiological significance. Anat Anz 48: 540-565
Gales N, Wallace G, Dickson J (1985) Pulmonary cryptococcosis in a striped dolphin (Stenella coeruleoalba). J Wildl Dis 21:443-446

Gargani L (2011) Lung ultrasound: a new tool for the cardiologist. Cardiovasc Ultrasound 9:6

Goerg C (2006) Lung masses. In: Schmidt G (ed) Differential diagnosis in ultrasound imaging: a teaching atlas. Georg Thieme Verlag, Stuttgart, p 469-488

Green RF (1972) Observations of the anatomy of some cetaceans and pinnipeds. In: Ridgway SH (ed) Mammals of the sea: biology and medicine. Charles C. Thomas Publishers, Springfield, IL, p 247-297

Greenwood AG, Taylor DC (1977) Clinical and pathological findings in dolphins in 1976. Aquat Mamm 5:34-39

Hecht S (2008) Thorax. In: Penninck D, D'Anjoy MA (eds) Atlas of small animal ultrasonography. Blackwell Publishing, Ames, IA, p 119-50

Howard EB, Britt JO, Matsumoto GK, Itahara R, Nagano CN (1983) Bacterial diseases. In: Howard EB (ed) Pathobiology of marine mammal diseases. CRC Press, Boca Raton, FL, p 69-118

- Inskeep W II, Gardiner CH, Harris RK, Dubey JP, Goldston RT (1990) Toxoplasmosis in Atlantic bottle-nosed dolphins (Tursiops truncatus). J Wildl Dis 26:377-382

Jensen ED, Lipscomb T, Van Bonn B, Miller G, Fradkin JM, Ridgway SH (1998) Disseminated histoplasmosis in an Atlantic bottlenose dolphin (Tursiops truncatus). J Zoo Wildl Med 29:456-460

Joseph BE, Cornell LH, Simpson JG, Migaki G, Griner L (1986) Pulmonary aspergillosis in three species of dolphin. Zoo Biol 5:301-308

Jung C, Bostedt H (2004) Thoracic ultrasonography technique in newborn calves and description of normal and pathological findings. Vet Radiol Ultrasound 45:331-335

Kuwamura M, Sawamoto O, Yamate J, Aoki M, Ohnishi Y, Kotani T (2007) Pulmonary vascular proliferation and lungworm (Stenurus ovatus) in a bottlenose dolphin (Tursiops truncatus). J Vet Med Sci 69:531-533

Larson MM (2009) Ultrasound of the thorax (noncardiac). Vet Clin Small Anim Pract 39:733-745

> Lichtenstein DA (2007) Ultrasound in the management of thoracic disease. Crit Care Med 35(Suppl):S250-S261

- Lichtenstein DA (2009) Ultrasound examination of the lungs in the intensive care unit. Pediatr Crit Care Med 10: 693-698

Lichtenstein D, Mézière G, Biderman P, Gepner A, Barré O (1997) The comet-tail artifact. An ultrasound sign of alveolar-interstitial syndrome. Am J Respir Crit Care Med 156:1640-1646

> Lipscomb TP, Kennedy S, Moffett D, Ford BK (1994a) Morbilliviral disease in an Atlantic bottlenose dolphin (Tursiops truncatus) from the Gulf of Mexico. J Wildl Dis 30: 572-576

Lipscomb TP, Schulman FY, Moffett D, Kennedy S (1994b) Morbilliviral disease in Atlantic bottlenose dolphins (Tursiops truncatus) from the 1987-1988 epizootic. J Wildl Dis 30:567-571

> Lipscomb TP, Kennedy S, Moffett D, Krafft A and others (1996) Morbilliviral epizootic in bottlenose dolphins of the Gulf of Mexico. J Vet Diagn Invest 8:283-290

> Louvet A, Bourgeois JM (2008) Lung ring-down artifact as a sign of pulmonary alveolar-interstitial disease. Vet Radiol Ultrasound 49:374-377

Lutmerding B, Johnson S, Ferrara S, Hill L, Ruiz C, EmoryGomez F, Jensen E (2010) Techniques in interventional 
radiology: case studies in marine mammal medicine. Proc Int Assoc Aquat Anim Med 41:74-76

- Makara M, Shimada A, Kawamura N, Murase T, Morita T (2007) Aspiration pneumonia as a cause of neonatal death in three captive bottlenose dolphins (Tursiops truncatus). J Vet Med Sci 69:325-327

Mattoon JS, Nyland TG (2002) Thorax. In: Nyland TG, Mattoon J (eds) Small animal diagnostic ultrasound, 2nd edn. WB Saunders, Philadelphia, PA, p 325-53

> Migaki G, Gunnels RD, Casey HW (1978) Pulmonary cryptococcosis in an Atlantic bottlenose dolphin (Tursiops truncatus). Lab Anim Sci 28:603-606

- Miller WG, Adams LG, Ficht TA, Cheville NF and others (1999) Brucella-induced abortions and infection in bottlenose dolphins (Tursiops truncatus). J Zoo Wildl Med 30:100-110

Miller WG, Padhye AA, van Bonn W, Jensen E, Brandt ME, Ridgway SH (2002) Cryptococcosis in a bottlenose dolphin (Tursiops truncatus) caused by Cryptococcus neoformans var. gattii. J Clin Microbiol 40:721-724

Moeller RB (2003) Pathology of marine mammals with special reference to infectious diseases. In: Vos JG, Bossart GD, Fournier M, O'Shea TJ (eds) Toxicology of marine mammals, Vol 3. Taylor \& Francis, New York, NY, p 6-8

Moore CL, Copel JA (2011) Point-of-care ultrasonography. N Engl J Med 364:749-757

> Nollens HH, Wellehan JF, Saliki JT, Caseltine SL, Jensen ED, Van Bonn W, Venn-Watson S (2008) Characterization of a parainfluenza virus isolated from a bottlenose dolphin (Tursiops truncatus). Vet Microbiol 128:231-242

Parsons EC, Overstreet RM, Jefferson TA (2001) Parasites from Indo-Pacific hump-backed dolphins (Sousa chinensis) and finless porpoises (Neophocaena phocaenoides) stranded in Hong Kong. Vet Rec 148:776-780

Piscitelli MA, McLellan WA, Rommel SA, Blum JE, Barco SG, Pabst DA (2010) Lung size and thoracic morphology in shallow- and deep-diving cetaceans. J Morphol 271: 654-673

Ramirez S, Lester GD, Roberts GR (2004) Diagnostic contribution of thoracic ultrasonography in 17 foals with Rhodococcus equi pneumonia. Vet Radiol Ultrasound 45: 172-176

Rantanen W (1981) Ultrasound appearance of normal lung borders and adjacent viscera in the horse. Vet Radiol 22: 217-219

$>$ Rantanen NW (1986) Diseases of the thorax. Vet Clin North Am Equine Pract 2:49-66

> Rantanen NW, Gage L, Paradis MR (1981) Ultrasonography as a diagnostic aid in pleural effusion in horses. Vet Radiol 22:211-216

> Reichle JK, Wisner ER (2000) Non-cardiac thoracic ultrasound in 75 feline and canine patients. Vet Radiol Ultrasound 41:154-162

Reidarson TH, Harrell JH, Rinaldi MG, McBain J (1998a) Bronchoscopic and serological diagnosis of Aspergillus fumigatus pulmonary infection in a bottlenose dolphin (Tursiops truncatus). J Zoo Wildl Med 29:451-455

Reidarson TH, Griner LH, Pappagianis D, McBain J (1998b) Coccidioidomycosis in a bottlenose dolphin. J Wildl Dis 34:629-631

> Ridgway SH, Scronce BL, Kanwisher J (1969) Respiration and deep diving in the bottlenose porpoise. Science 166: 1651-1654

Sartori S, Tombesi P (2010) Emerging roles for transthoracic ultrasonography in pleuropulmonary pathology. World J
Radiol 2:83-90

Saunders HM, Keith D (2004) Thoracic imaging. In: King LG (ed) Textbook of respiratory diseases in dogs and cats. WB Saunders, Philadelphia, PA, p 72-93

Schulman FY, Lipscomb TP, Moffett D, Krafft AE and others (1997) Histological, immunohistochemical, and polymerase chain reaction studies of bottlenose dolphins from the 1987-1988 United States Atlantic coast epizootic. Vet Pathol 34:288-295

Simpson JG, Gardner MB (1972) Comparative microscopic anatomy of selected marine mammals. In: Ridgway $\mathrm{SH}$ (ed) Mammals of the sea: biology and medicine. Charles C. Thomas Publishers, Springfield, IL, p 298-418

St. Leger JA, Begeman L, Fleetwood M, Frasca S and others (2009) Comparative pathology of nocardiosis in marine mammals. Vet Pathol 46:299-308

Stefanidis K, Dimopoulos S, Nanas S (2011) Basic principles and current applications of lung ultrasonography in the intensive care unit. Respirology 16:249-256

Stone LR (1990) Diagnostic ultrasound of marine mammals. In: Dierauf LA (ed) CRC handbook of marine mammal medicine: health, disease, and rehabilitation. CRC Press, Boca Raton, FL, p 243-247

Stowater JL, Lamb CR (1989) Ultrasonography of noncardiac thoracic diseases in small animals. J Am Vet Med Assoc 195:514-520

Sweeney JC, Ridgway SH (1975) Common diseases of small cetaceans. J Am Vet Med Assoc 167:533-540

Tangredi BP, Medway W (1980) Post-mortem isolation of Vibrio alginolyticus from an Atlantic white-sided dolphin (Lagenorhynchus acutus). J Wildl Dis 16:329-331

> Targhetta R, Chavagneux R, Bourgeois JM, Dauzat M, Balmes P, Pourcelot L (1992) Sonographic approach to diagnosing pulmonary consolidation. J Ultrasound Med 11:667-672

Tidwell AS (1998) Ultrasonography of the thorax (excluding the heart). Vet Clin North Am Small Anim Pract 28: 993-1015

Van Bonn W, Jensen ED (2001) Percutaneous thoracic fine needle aspiration biopsy in dolphins. Proc Int Assoc Aquat Anim Med 32:69-72

- Venn-Watson S, Rivera R, Smith CR, Saliki JT and others (2008) Exposure to novel Parainfluenza virus and clinical relevance in two bottlenose dolphin (Tursiops truncatus) populations. Emerg Infect Dis 14:397-405

Venn-Watson SK, Jensen ED, Ridgway SH (2011a) Evaluation of population health among bottlenose dolphins (Tursiops truncatus) at the United States Navy Marine Mammal Program. J Am Vet Med Assoc 238:356-360

> Venn-Watson S, Smith CR, Gomez F, Jensen ED (2011b) Physiology of aging among healthy, older bottlenose dolphins (Tursiops truncatus): comparisons with aging humans. J Comp Physiol B 181:667-680

Venn-Watson S, Daniels R, Smith CR (2012) Thirty year retrospective evaluation of pneumonia in a bottlenose dolphin Tursiops truncatus population. Dis Aquat Org 99: 237-242

Volpicelli G, Mussa A, Garofalo G, Cardinale L and others (2006) Bedside lung ultrasound in the assessment of alveolar-interstitial syndrome. Am J Emerg Med 24: 689-696

Wislocki GB (1929) On the structure of the lungs of the porpoise (Tursiops truncatus). Am J Anat 44:47-77

Woodard JC, Zam SG, Caldwall DK, Caldwell MC (1969) Some parasitic diseases of dolphins. Pathol Vet 6:257-272 